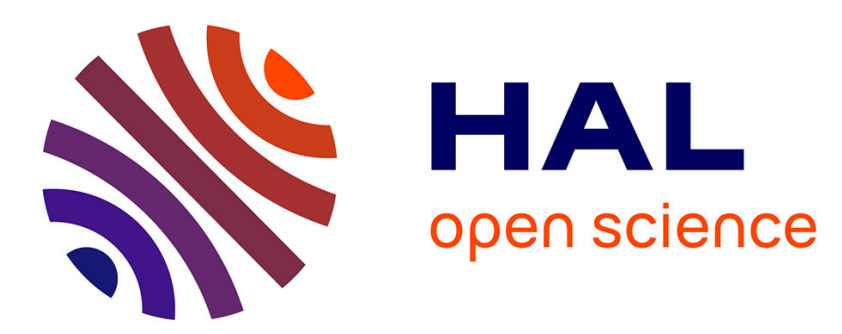

\title{
Étude des transferts d'orientation et d'alignement dans la fluorescence sensibilisée mercure-cadmium et mercure-zinc
}

\author{
G. Cremer, B. Cheron
}

\section{- To cite this version:}

G. Cremer, B. Cheron. Étude des transferts d'orientation et d'alignement dans la fluorescence sensibilisée mercure-cadmium et mercure-zinc. Journal de Physique Lettres, 1978, 39 (22), pp.419-423. 10.1051/jphyslet:019780039022041900 . jpa-00231533

\section{HAL Id: jpa-00231533 https://hal.science/jpa-00231533}

Submitted on 1 Jan 1978

HAL is a multi-disciplinary open access archive for the deposit and dissemination of scientific research documents, whether they are published or not. The documents may come from teaching and research institutions in France or abroad, or from public or private research centers.
L'archive ouverte pluridisciplinaire HAL, est destinée au dépôt et à la diffusion de documents scientifiques de niveau recherche, publiés ou non, émanant des établissements d'enseignement et de recherche français ou étrangers, des laboratoires publics ou privés. 


\title{
ÉTUDE DES TRANSFERTS D'ORIENTATION ET D'ALIGNEMENT DANS LA FLUORESCENCE SENSIBILISÉE MERCURE-CADMIUM ET MERCURE-ZINC
}

\author{
G. CREMER et B. CHERON
}

Laboratoire de Spectroscopie atomique $\left({ }^{*}\right)$, Université de Caen, 14032 Caen Cedex, France

(Reçu le 13 juillet 1978, révisé le 2 octobre 1978, accepté le 3 octobre 1978)

$$
\begin{aligned}
& \text { Résumé. - Les auteurs étudient le transfert d'excitation lors des collisions : } \\
& \text { et } \begin{aligned}
\operatorname{Hg}\left(6{ }^{1} \mathrm{P}_{1}\right)+\mathrm{Zn}\left(4{ }^{1} \mathrm{~S}_{0}\right) \rightarrow \mathrm{Hg}\left(6{ }^{1} \mathrm{~S}_{0}\right)+\mathrm{Zn}\left(5{ }^{3} \mathrm{~S}_{1}\right)+0,049 \mathrm{eV} \\
\operatorname{Hg}\left(6{ }^{1} \mathrm{P}_{1}\right)+\mathrm{Cd}\left({ }^{1} \mathrm{~S}_{0}\right) \rightarrow \mathrm{Hg}\left(6{ }^{1} \mathrm{~S}_{0}\right)+\mathrm{Cd}\left(6{ }^{3} \mathrm{~S}_{1}\right)+0,32 \mathrm{eV}
\end{aligned}
\end{aligned}
$$

Au moyen de l'effet Hanle, ils mesurent le taux de transfert d'alignement pour la collision $\mathrm{Hg}-\mathrm{Zn}$ : $\alpha_{\mathbf{Z n}}^{(2)}=-0,19 \pm 0,03$, ainsi que les taux de transfert d'orientation : $\alpha_{\mathbf{Z n}}^{(1)} \leqslant 0,01 ; \alpha_{\mathrm{Cd}}^{(1)} \leqslant 0,03$.

Abstract. - Excitation transfer resulting from the collisions :

and

$$
\mathrm{Hg}\left(6{ }^{1} \mathrm{P}_{1}\right)+\mathrm{Zn}\left(4{ }^{1} \mathrm{~S}_{0}\right) \rightarrow \mathrm{Hg}\left(6{ }^{1} \mathrm{~S}_{0}\right)+\mathrm{Zn}\left(5{ }^{3} \mathrm{~S}_{1}\right)+0.049 \mathrm{eV}
$$

$$
\mathrm{Hg}\left(6{ }^{1} \mathrm{P}_{1}\right)+\mathrm{Cd}\left(5{ }^{1} \mathrm{~S}_{0}\right) \rightarrow \mathrm{Hg}\left(6{ }^{1} \mathrm{~S}_{0}\right)+\mathrm{Cd}\left(6{ }^{3} \mathrm{~S}_{1}\right)+0.32 \mathrm{eV}
$$

have been studied by means of the Hanle effect technique. The alignment transfer rate for $\mathrm{Hg}-\mathrm{Zn}$ collisions was found to be $: \alpha_{\mathbf{Z n}}^{(2)}=-0.19 \pm 0.03$. Orientation transfer rates for both processes are $: \alpha_{\mathrm{Zn}}^{(1)} \leqslant 0.01, \alpha_{\mathrm{Cd}}^{(1)} \leqslant 0.03$.

1. Introduction. - $\mathrm{Si}$ on éclaire un mélange de vapeurs de mercure et de cadmium (ou de zinc) par la raie de résonance $\lambda=185 \mathrm{~nm}\left(6{ }^{1} \mathrm{~S}_{0}-6{ }^{1} \mathrm{P}_{1}\right) \mathrm{du}$ mercure, on observe en plus de la réémission de cette même raie (résonance optique), une fluorescence de la vapeur de cadmium (ou de zinc) correspondant aux diverses transitions de la figure 1 . C'est le phénomène de fluorescence sensibilisée, qui résulte du transfert d'excitation, lors d'une collision de seconde espèce, et que l'on peut représenter par les équations :

$$
\begin{aligned}
\mathrm{Hg}\left(6{ }^{1} \mathrm{P}_{1}\right) & +\mathrm{Cd}\left(5{ }^{1} \mathrm{~S}_{0}\right) \rightarrow \\
& \rightarrow \mathrm{Hg}\left(6{ }^{1} \mathrm{~S}_{0}\right)+\mathrm{Cd}\left(6{ }^{3} \mathrm{~S}_{1}\right)+0,32 \mathrm{eV}, \\
\mathrm{Hg}\left(6{ }^{1} \mathrm{P}_{1}\right) & +\mathrm{Zn}\left(4{ }^{1} \mathrm{~S}_{0}\right) \rightarrow \\
\rightarrow & \mathrm{Hg}\left(6{ }^{1} \mathrm{~S}_{0}\right)+\mathrm{Zn}\left(5{ }^{3} \mathrm{~S}_{1}\right)+0,049 \mathrm{eV} .
\end{aligned}
$$

Ce phénomène a déjà fait l'objet de nombreux travaux expérimentaux, portant sur la détermination des sections efficaces de transfert d'excitation [1], [2], [3] et sur la mesure du transfert de polarisation [3], [4].

(*) Laboratoire associé au C.N.R.S. $\mathrm{n}^{\circ} 19$.
Au cours d'une collision de type (1) ou (2), une partie de l'alignement des atomes de mercure est transférée aux atomes de cadmium ou de zinc. Saintout [4] a mesuré le transfert d'alignement pour la collision (1) et a obtenu :

$$
\alpha_{\mathrm{Cd}}^{(2)}=-(0,20 \pm 0,05)
$$

Nous présentons les résultats de l'étude du transfert d'alignement, au cours de la collision (2), et nous donnons une limite supérieure du taux de transfert d'orientation pour les collisions (1) et (2).

2. Dispositif expérimental (Fig. 2). - Une cellule cubique de $3 \mathrm{~cm}$ d'arête, contenant le mélange de vapeurs de mercure et cadmium (ou zinc) naturels est éclairée par un faisceau lumineux $(\lambda=185 \mathrm{~nm})$ non polarisé (expérience de transfert d'alignement) ou polarisé circulairement (expériences de transfert d'orientation), se propageant suivant la direction $O x^{\prime}$ (Fig. 2) et provenant d'une lampe à vapeur de mercure excitée dans une cavité micro-onde. Un courant gazeux produit par évaporation d'azote liquide refroidit la lampe de façon à éviter le renversement de la 


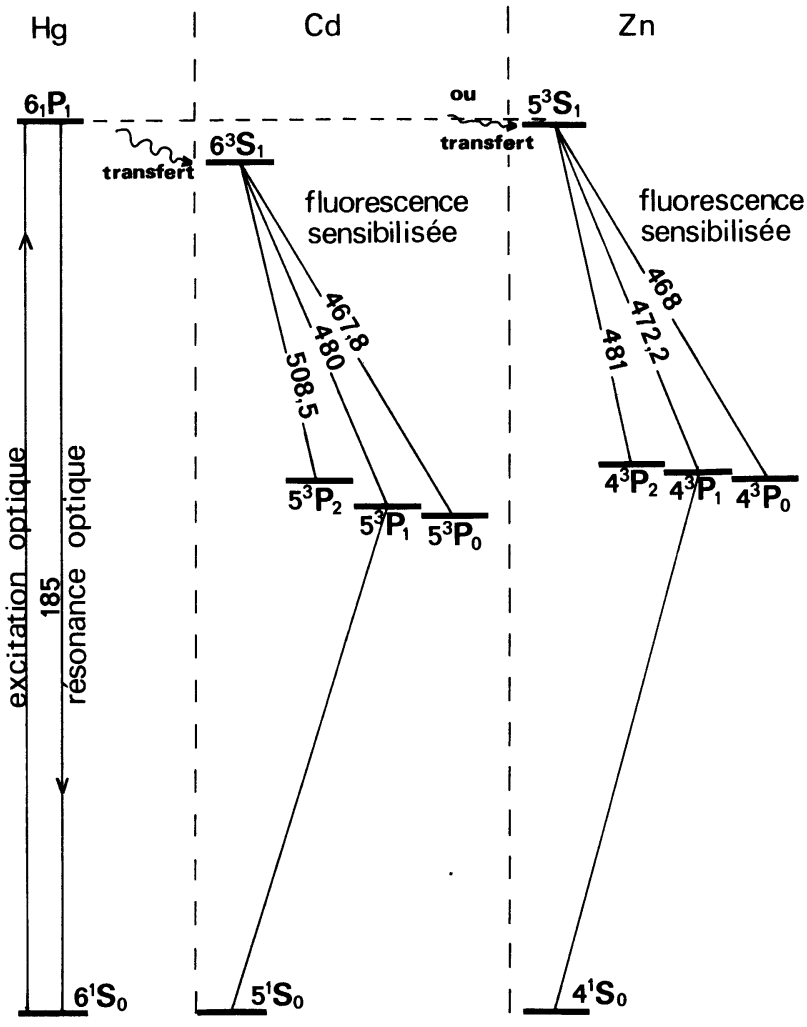

Fig. 1. - Premiers niveaux d'énergie des atomes de mercure, cadmium et zinc. Les longueurs d'onde sont exprimées en nanomètre.

[First energy levels of $\mathrm{Hg}, \mathrm{Cd}$ and $\mathrm{Zn}$. Wave lengths are in nanometers.]

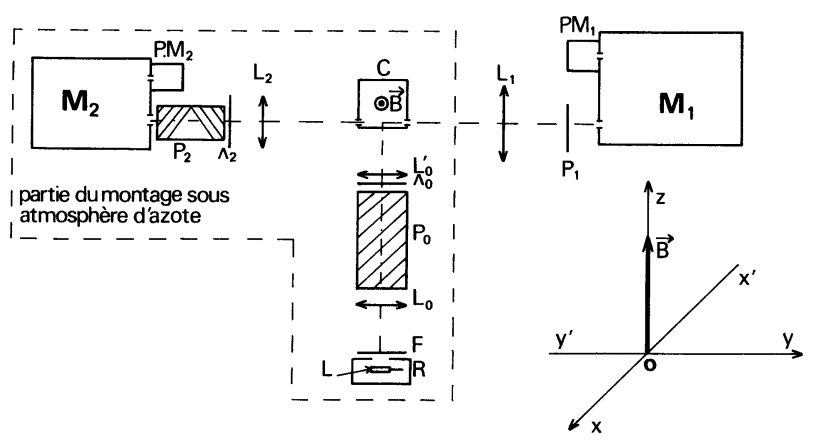

Fig. 2. - Schéma du montage expérimental. $C$ cellule; $M_{1}$ monochromateur détectant la fluorescence sensibilisée; $\mathbf{M}_{2}$ monochromateur détectant la fluorescence directe à $185 \mathrm{~nm}$; L lampe à mercure naturel, dans une cavité résonnante $\mathrm{R} ; \mathrm{F}$ filtre à $185 \mathrm{~nm}$; $\mathrm{L}_{0}, \mathrm{~L}_{0}^{\prime}, \mathrm{L}_{1}, \mathrm{~L}_{2}$ lentilles; $\mathrm{PM}_{1}, \mathrm{PM}_{2}$ photomultiplicateurs. Pour l'étude de l'orientation : $\Lambda_{0}$ lame $\lambda / 4$ à $185 \mathrm{~nm}$ par compression; $\Lambda_{2}$ lame $\lambda / 4$ cristalline; $P_{0}$ et $P_{2}$ polariseurs à piles de glaces $P_{1}$ polariseur circulaire HNCP 37. Pour l'étude de l'alignement : $\Lambda_{0}, \mathrm{P}_{0}, \Lambda_{2}$ supprimés et $\mathrm{P}_{1}$ remplacé par un polariseur linéaire HN38.

[Experimental set up. $\mathrm{C}$ cell; $\mathbf{M}_{1}$ monochromator for sensitized fluorescence detection; $\mathbf{M}_{2}$ monochromator for direct fluorescence detection at $185 \mathrm{~nm}$; L natural $\mathrm{Hg}$ lamp in the resonant cavity $\mathrm{R}$; $F$ filter at $185 \mathrm{~nm} ; \mathrm{L}_{0}, \mathrm{~L}_{0}^{\prime}, \mathrm{L}_{1}, \mathrm{~L}_{2}$ lenses; $\mathrm{PM}_{1}, \mathrm{PM}_{2}$ photomultipliers. For orientation study : $\Lambda_{0}, \lambda / 4$ stressed plate at $185 \mathrm{~nm}$; $\Lambda_{2}, \lambda / 4$ plate at $185 \mathrm{~nm} ; \mathrm{P}_{0}$ and $\mathrm{P}_{2}$ linear polarizers $; \mathrm{P}_{1}$ circular polarizer HNCP 37. For alignment study : $\Lambda_{0}, \mathrm{P}_{0}, \Lambda_{2}$ suppressed and $P_{1}$ replaced by a linear polarizer $H N 38$.] raie $185 \mathrm{~nm}$. Le contrôle et la mesure des pressions de vapeurs de mercure et de cadmium (ou de zinc) sont délicats, car la cellule n'est pas scellée mais reliée à un bâti de pompage, et aussi parce qu'il se forme, dans les deux cas, un amalgame. Toutefois, au cours des expériences qui durent en général plusieurs heures, la tension de vapeur du mercure est plus stable dans le cas de l'étude du couple Hg-Zn, que dans le cas du couple $\mathrm{Hg}-\mathrm{Cd}$ : il en résulte donc une moins grande dispersion des points sur la courbe permettant de calculer le taux de transfert $\alpha_{\mathrm{Zn}}^{(2)}$ et par conséquent une estimation de $\alpha_{\mathrm{Zn}}^{(2)}$ plus précise que celle de $\alpha_{\mathrm{Cd}}^{(2)}$. Un premier monochromateur à réseau isole la fluorescence sensibilisée (F.S.) émise dans la direction $\mathrm{O} y$ $\left(\lambda_{\mathrm{Cd}}=480 \mathrm{~nm}\right.$ ou $\left.\lambda_{\mathrm{zn}}=472,2 \mathrm{~nm}\right)$ et un second isole la raie de résonance $(\lambda=185 \mathrm{~nm})$ du mercure (F.D.) émise dans la direction $\mathrm{O} y^{\prime}$. Le champ magnétique B a pour direction l'axe $\mathrm{O} z$ choisi vertical, et il est produit par une paire de bobines en position de Helmholtz. La détection du signal d'effet Hanle nécessite l'emploi d'une technique d'accumulation dans les mémoires d'un analyseur multicanal.

3. Résultats. - 3.1 ETUDE DU TRANSFERT D'AliGNEMENT : HG-ZN. - Avec le dispositif expérimental de la figure 2 , excitation en lumière naturelle et polariseur linéaire à la détection, la vapeur de mercure est alignée dans l'état $6{ }^{1} \mathrm{P}_{1}$ : on observe sur la fluorescence directe du mercure détectée dans la direction $\mathrm{O} y^{\prime}$ avec une polarisation $\mathrm{e}_{\mathrm{D}}=\mathbf{i}$, un signal d'effet Hanle caractéristique du niveau $6{ }^{1} \mathrm{P}_{1}$ (durée de vie $\tau_{\mathrm{A}}=1,36 \times 10^{-9} \mathrm{~s}$ ). En fluorescence sensibilisée on observe alors sur chacune des raies du triplet visible détectée dans la direction $\mathrm{O} y$ avec une polarisation $\mathbf{e}_{\mathrm{D}}=\mathbf{i}$, un signal d'effet Hanle (Fig. 3). Nous constatons que : (i) La largeur du signal d'effet Hanle est caractéristique de l'alignement des atomes de zinc dans l'état $5^{3} \mathrm{~S}_{1}$ de durée de vie $\tau_{\mathrm{B}}=8,15 \times 10^{-9} \mathrm{~s}$. (ii) Pour les conditions géométriques d'excitation et de détection fixées, le taux de polarisation de la lumière de fluorescence du mercure est une fonction décroissante de la pression de vapeur de mercure (diffusion multiple). Or, si on fait varier cette pression de vapeur de mercure, on constate que le taux de polarisation de la fluorescence sensibilisée pour une raie donnée du triplet visible est une fonction croissante du taux de polarisation de la fluorescence directe du mercure (Fig. 4). La figure 4 représente le taux de polarisation $\left(P_{\mathrm{Zn}}\right)$ de la raie $472,2 \mathrm{~nm}$, en fonction du taux de polarisation $\left(P_{\mathrm{Hg}}\right)$ de la raie $185 \mathrm{~nm}$. Ces quantités sont définies comme le rapport de l'amplitude de la variation du signal lorsque le champ magnétique $B$ varie de 0 à une valeur infinie $(I(\infty)$ - $I(0))$ à l'amplitude de ce même signal lorsque le champ est infini $(I(\infty))$. Lorsque la pression de vapeur de mercure tend vers 0 , l'expérience montre que $P_{\mathrm{Hg}}$ tend vers $53 \pm 2 \%$ et nous obtenons par une simple extrapolation linéaire sur la figure 4 le 


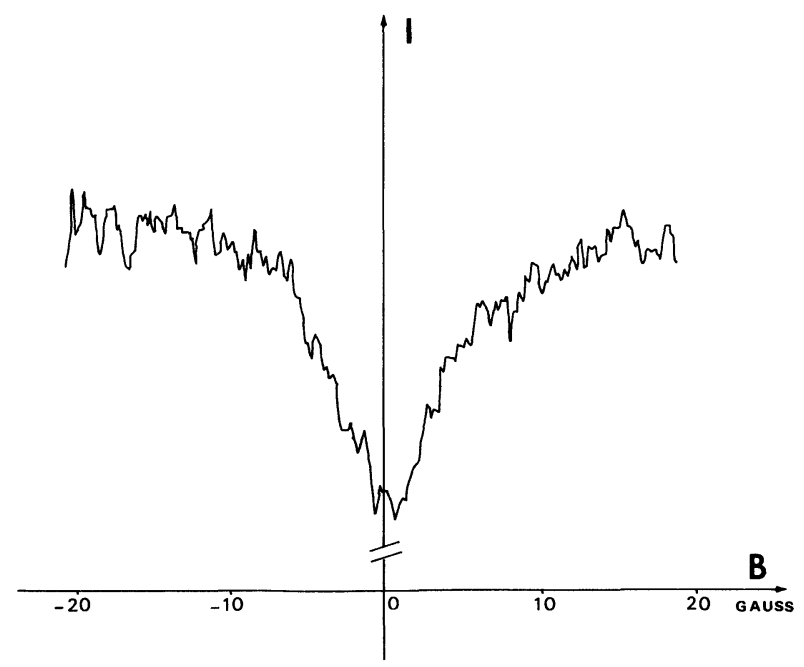

FIG. 3. - Intensité de la fluorescence sensibilisée : transition $4{ }^{3} \mathrm{P}_{1}-5{ }^{3} \mathrm{~S}_{1}, \lambda=472,2 \mathrm{~nm}$ du zinc, détectée dans la direction ()$y$, avec une polarisation $\mathbf{e}_{\mathrm{D}}=\mathbf{i}$, en fonction du champ magnétique $B$. Durée d'accumulation : 5 h $30 \mathrm{~min}$.

[Sensitized fluorescence intensity versus magnetic field intensity $B$ for the transition $4{ }^{3} \mathrm{P}_{1}-5{ }^{3} \mathrm{~S}_{1}(\lambda=472.2 \mathrm{~nm})$ of $\mathrm{Zn}$ detected in the $\mathrm{O} y$ direction, with a polarization $\mathbf{e}_{\mathrm{D}}=\mathbf{i}$. Accumulation time $5 \mathrm{~h} 30 \mathrm{~min}$.]

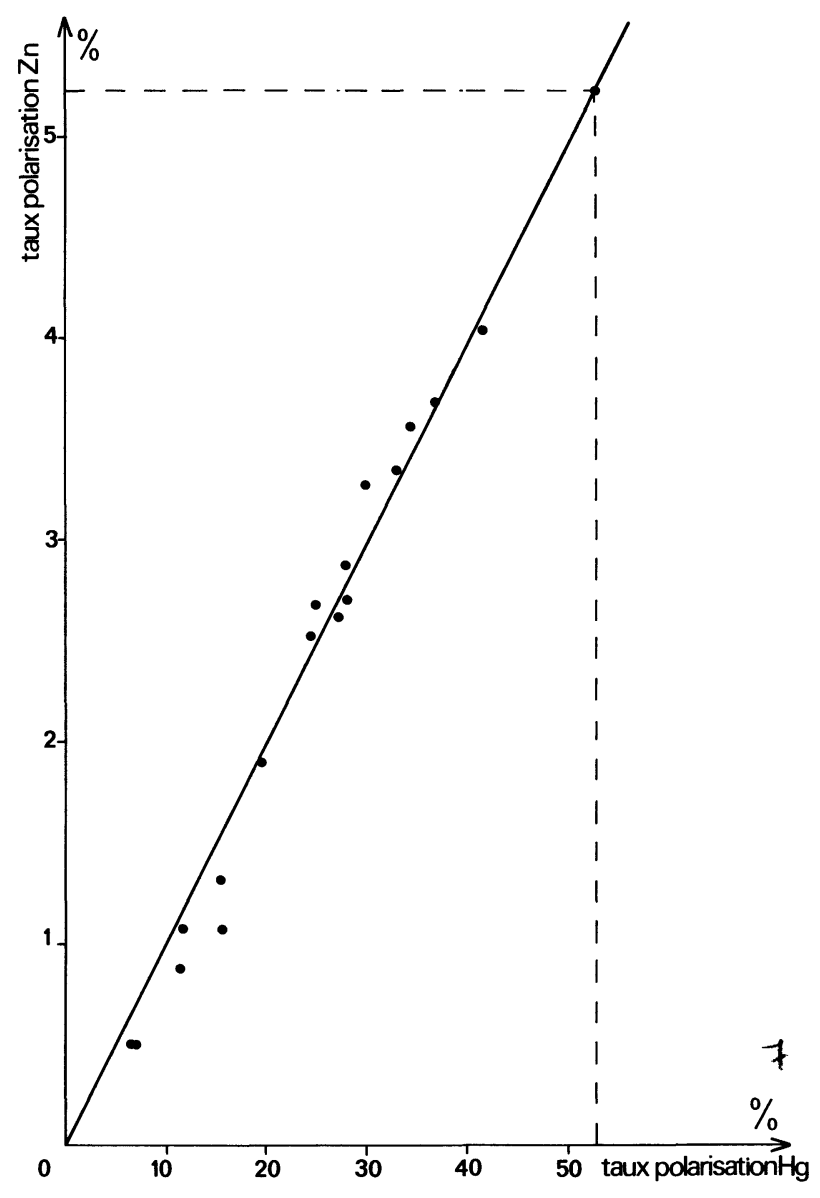

Fig. 4. - Transfert d'alignement $\mathrm{Hg}-\mathrm{Zn}: P_{\mathrm{Zn}}=f\left(P_{\mathrm{Hg}}\right)$.

[Alignment transfer $\mathrm{Hg}-\mathrm{Zn}: P_{\mathrm{Zn}}=f\left(P_{\mathrm{Hg}}\right)$.] taux de polarisation maximum de la fluorescence sensibilisée $P_{\mathrm{Zn}}=5,25 \%$. Une méthode d'extrapolation plus réaliste décrite dans la référence [3] donnerait un résultat très voisin en raison de la dispersion des points expérimentaux. (iii) Le transfert d'alignement se fait avec changement de signe comme dans le cas du cadmium : la courbe d'effet Hanle obtenue en fluorescence sensibilisée sur la raie $468 \mathrm{~nm}$ et celle que l'on obtient dans la fluorescence directe du mercure $(\lambda=185 \mathrm{~nm})$ détectée avec la même polarisation sont de sens opposés.

En l'absence de tout phénomène dépolarisant et pour des isotopes pairs, le signal théorique détecté en F.S. sur la raie 472,2 $\mathrm{nm}$ est proportionnel à [3] :

$$
\begin{aligned}
I_{\mathrm{B}} & =\frac{2}{3}+\frac{\alpha^{(2)}}{12}+\frac{\alpha^{(2)}}{4} \times \\
& \times \frac{\Delta_{\mathrm{B}}}{\Delta_{\mathrm{A}}-\Delta_{\mathrm{B}}}\left[\frac{\Delta_{\mathrm{A}}}{\Delta_{\mathrm{B}}} \cdot \frac{\Delta_{\mathrm{B}}^{2}}{\Delta_{\mathrm{B}}^{2}+4 B^{2}}-\frac{\Delta_{\mathrm{A}}^{2}}{\Delta_{\mathrm{A}}^{2}+4 B^{2}}\right]
\end{aligned}
$$

où $\alpha^{(2)}$ est le rapport de la section efficace de transfert d'alignement à la section efficace de transfert de population et où $\Delta_{\mathrm{A}}$ et $\Delta_{\mathrm{B}}$ sont les largeurs des courbes d'effet Hanle obtenues en fluorescence directe sur les niveaux $\mathrm{Hg}\left(6{ }^{1} \mathrm{P}_{1}\right)$ et $\mathrm{Zn}\left(5{ }^{3} \mathrm{~S}_{1}\right)$ respectivement. Pour les isotopes pairs $\Delta_{\mathrm{A}}=82,4$ gauss et $\Delta_{\mathrm{B}}=6,98$ gauss. La courbe résultante est donc peu différente d'une lorentzienne de largeur à mi-hauteur $\Delta_{\mathrm{B}}$. Le rapport signal sur bruit de la courbe de la figure 3 n'est pas suffisant pour préciser ce point. La largeur à mi-hauteur des courbes observées varie entre 6,8 et 7,6 G. Le taux de polarisation théorique $P_{\mathrm{zn}}$ est alors :

$$
P_{\mathrm{Zn}}=\frac{-0,375 \alpha^{(2)}}{1+0,125 \alpha^{(2)}} .
$$

On peut donc déduire $\alpha^{(2)}$ de la mesure de $P_{\mathrm{Zn}}$. Cependant des corrections liées aux imperfections du montage et à l'utilisation d'éléments naturels contenant des isotopes impairs, sont nécessaires. L'effet de la structure isotopique peut être évalué si on connaît la distribution spectrale de la lampe excitatrice et moyennant une hypothèse sur le comportement du spin nucléaire des isotopes impairs lors de la collision. Il ne semble pas déraisonnable de supposer une excitation uniforme des différentes composantes hyperfines du mercure. D'autre part, nous supposons que lors de la collision, le spin nucléaire n'est pas affecté et que seules les grandeurs électroniques sont transférées de l'atome de mercure à l'atome de zinc. Nous obtenons alors :

$$
P_{\mathrm{Zn}}=\frac{-0,295 \alpha^{(2)}}{1+0,10 \alpha^{(2)}}
$$

ainsi que $P_{\mathrm{Hg}}=62,5 \%$. Cette dernière valeur est à comparer avec la valeur expérimentale $(53 \pm 2 \%)$. L'écart relatif $\varepsilon$ est de $18 \%$. Il peut provenir : (i) De l'ouverture des faisceaux à l'excitation ainsi que de 
l'hypothèse simplificatrice concernant le spectre émis par la lampe à mercure. Ces deux facteurs influencent la polarisation de la F.D. et la F.S. (ii) De la surestimation de la qualité du polariseur utilisé à la détection de la F.D. et de la faible biréfringence des faces de la cellule, l'effet dépolarisant de ce dernier défaut étant plus important en F.D. (185 nm) qu'en F.S. (472 nm). Nous admettons que l'écart observé entre les valeurs théorique et expérimentale du taux de polarisation du mercure est lié au fait qu'il ne nous est pas possible d'évaluer avec précision toutes les imperfections du montage à l'excitation et à la détection. Dans ces conditions, nous corrigeons arbitrairement la valeur de $P_{\mathrm{Zn}}$ par le facteur multiplicatif $1+(\varepsilon / 2)$ soit 1,09 . On en déduit alors $\alpha^{(2)}$ à partir de la formule (5)

$$
\alpha^{(2)}=-(0,19 \pm 0,03) \text {. }
$$

Ce résultat montre que, comme dans le cas du cadmium, le transfert d'alignement se fait avec un changement de signe.

3.2 ETUde DU TRANSFERT D'ORIENTATION HG-CD ET HG-ZN. - Nous excitons la vapeur de mercure, en lumière polarisée circulairement, à l'aide d'un polariseur à pile de glaces suivi d'une lame quart d'onde par compression. A la détection, pour le mercure $(\lambda=185 \mathrm{~nm})$, nous utilisons un système analogue pour mesurer le taux d'orientation de la vapeur ; pour le cadmium $(\lambda=480 \mathrm{~nm})$ et pour le zinc $(\lambda=472,2 \mathrm{~nm})$ nous disposons de polariseurs circulaires du type Polaroïd HNCP 37 droits ou gauches. Le signal d'effet Hanle théorique, attendu pour la fluorescence sensibilisée, est :

$$
\begin{aligned}
& I(B)=\frac{\varepsilon \varepsilon^{\prime}}{4} \alpha^{(1)} \frac{\Delta_{\mathrm{B}}}{\Delta_{\mathrm{A}}-\Delta_{\mathrm{B}}}\left[\frac{\Delta_{\mathrm{A}}}{\Delta_{\mathrm{B}}} \cdot \frac{B \Delta_{\mathrm{B}}}{\Delta_{\mathrm{B}}^{2}+B^{2}}-\frac{B \Delta_{\mathrm{A}}}{\Delta_{\mathrm{A}}^{2}+B^{2}}\right]+ \\
& +\frac{1}{16} \alpha^{(2)} \frac{\Delta_{\mathrm{B}}}{\Delta_{\mathrm{A}}-\Delta_{\mathrm{B}}}\left[\frac{\Delta_{\mathrm{A}}}{\Delta_{\mathrm{B}}} \cdot \frac{\Delta_{\mathrm{B}}^{2}}{\Delta_{\mathrm{B}}^{2}+4 B^{2}}-\frac{\Delta_{\mathrm{A}}^{2}}{\Delta_{\mathrm{A}}^{2}+4 B^{2}}\right] \\
& +\frac{1}{3}-\frac{\alpha^{(2)}}{48}
\end{aligned}
$$

où $\varepsilon= \pm 1, \varepsilon^{\prime}= \pm 1$ selon que l'on excite ou détecte en lumière circulaire gauche ou droite. $\alpha^{(1)}$ est le taux de transfert d'orientation, c'est-à-dire le rapport de la section efficace de transfert d'orientation à la section efficace de transfert de population. Le premier terme caractérise l'orientation (fonction impaire du champ $B$ ) et le second l'alignement (fonction paire du champ $B$ ). Nous définissons le taux de polarisation d'orientation comme le rapport de l'amplitude de la variation du terme impair à l'intensité du signal lorsque $B \rightarrow \infty$. Si on compare les courbes expérimentales obtenues au signal théorique attendu, on obtient dans tous les cas un signal qui caractérise l'alignement de la vapeur de $\mathrm{Cd}$ (Fig. 5) ou de la vapeur de $\mathrm{Zn}$ (Fig. 6) dans les niveaux $6{ }^{3} \mathrm{~S}_{1}$ et $5{ }^{3} \mathrm{~S}_{1}$ respectivement. Cela signifie que $\alpha^{(1)}$ est très inférieur à $\alpha^{(2)}$. Dans le cas du zinc, on observe une légère dissy-

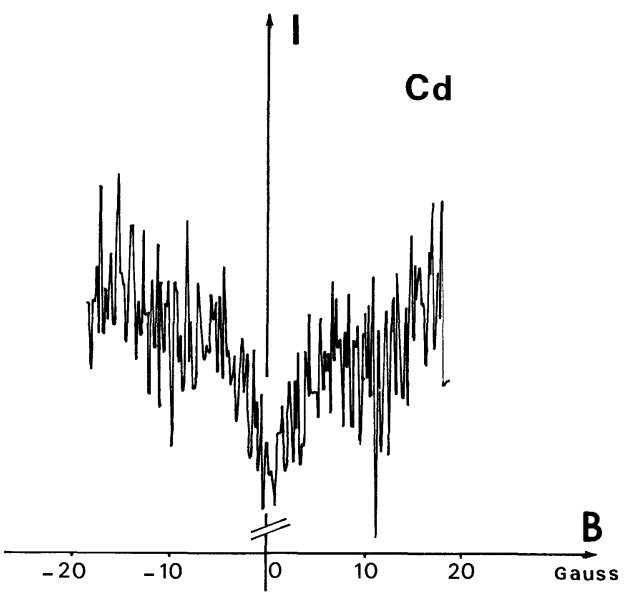

FIG. 5. - Transfert d'orientation $\mathrm{Hg}-\mathrm{Cd}$ : excitation en lumière circulaire et détection en lumière circulaire. Observation de l'alignement (taux de polarisation du cadmium $1,2 \%$ avec un taux de $30 \%$ pour le mercure en orientation). Durée d'accumulation : $20 \mathrm{~h}$.

[Orientation transfer $\mathrm{Hg}-\mathrm{Cd}$ : excitation and detection in circularly polarized light. Observation of alignment (polarization rate of Cd $1.2 \%$ with a polarization rate of $30 \%$ for the orientation of mercury). Accumulation time : $20 \mathrm{~h}$.]

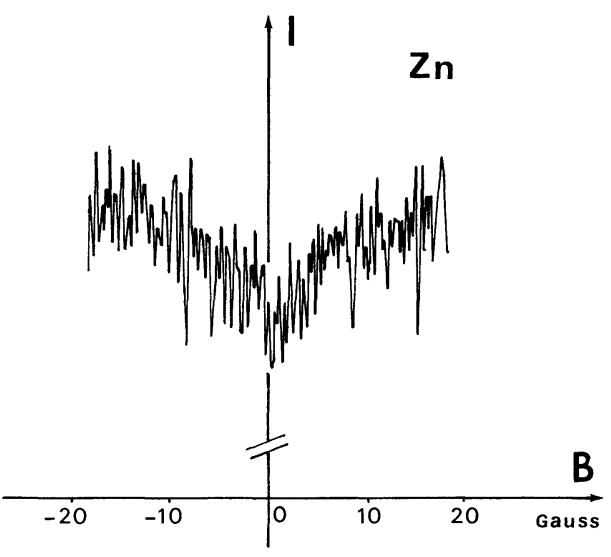

FIG. 6. - Transfert d'orientation $\mathrm{Hg}-\mathrm{Zn}$ : excitation en lumière circulaire et détection en lumière circulaire. Observation de l'alignement (taux de polarisation du zinc $\simeq 1 \%$ avec un taux de polarisation de $28,5 \%$ pour le mercure en orientation). Durée d'accumulation $25 \mathrm{~h}$.

[Orientation transfer $\mathrm{Hg}-\mathrm{Zn}$ : excitation and detection in circularly polarized light. Observation of alignment (polarization rate of $\mathrm{Zn} \simeq 1 \%$ with a polarization rate of $28.5 \%$ for the orientation of $\mathrm{Hg}$ ). Accumulation time : $25 \mathrm{~h}$.]

métrie sur les courbes, différente selon que l'on détecte en lumière circulaire droite ou gauche. En faisant la différence de 2 courbes de chaque type, nous éliminons théoriquement l'alignement et doublons le signal d'orientation. Expérimentalement, nous observons que ce signal est complètement noyé dans le bruit et nous pouvons affirmer que le taux de polarisation d'orientation sur le zinc est certainement inférieur à $1,8 \times 10^{-3}$ pour un taux de polarisation sur le mercure de l'ordre de $29 \%$. De même, 
dans le cas du cadmium, nous estimons que le taux de polarisation d'orientation est inférieur à $6 \times 10^{-3}$ pour un taux de polarisation sur le mercure de l'ordre de $29 \%$. Après évaluation des imperfections du montage, ceci nous conduit aux estimations suivantes :

$$
\alpha_{\text {Cd }}^{(1)} \leqslant 0,03 ; \quad \alpha_{\mathbf{Z n}_{\mathbf{n}}}^{(1)} \leqslant 0,01 .
$$

Il semble donc, que contrairement à l'alignement, l'orientation ne se transfère pas dans la collision envisagée. Il y a lieu de se demander s'il est possible d'orienter les niveaux $5{ }^{3} \mathrm{~S}_{1}$ du zinc et $6{ }^{3} \mathrm{~S}_{1}$ du cadmium en fluorescence directe. L'expérience a été faite sur le montage, pour le cadmium, avec des lampes Osram en excitation par échelons. Le niveau $5{ }^{3} \mathrm{P}_{1}$ (Cd) est peuplé de façon isotrope par absorption de la raie $326,1 \mathrm{~nm}$ (excitation en lumière naturelle). Le niveau $6{ }^{3} \mathrm{~S}_{1}$ est peuplé par absorption de la raie $480 \mathrm{~nm}$ polarisée circulairement, à l'aide d'un polariseur $\mathrm{HNCP}$ 37. On a pu détecter l'orientation sur les différentes raies du triplet visible : les courbes obtenues ont une largeur caractéristique du niveau $6{ }^{3} \mathrm{~S}_{1}$, soit de l'ordre de 12,9 gauss (largeur attendue 12,4 gauss). Ce résultat prouve d'une part que dans les conditions de pressions de vapeur de $\mathrm{Hg}$ et de Cd utilisées, l'orientation des atomes $\mathrm{Cd}\left(6{ }^{3} \mathrm{~S}_{1}\right)$ créée optiquement est conservée et d'autre part que le système de détection est capable de mettre en évidence cette orientation.

4. Conclusion. - Ces résultats diffèrent notablement de ceux obtenus dans le cas du transfert :

$$
\begin{aligned}
\operatorname{Hg}\left(6{ }^{3} P_{1}\right) & +\mathrm{Cd}\left(5{ }^{1} \mathrm{~S}_{0}\right) \rightarrow \\
& \rightarrow \operatorname{Hg}\left(6{ }^{1} \mathrm{~S}_{0}\right)+\mathrm{Cd}\left(5{ }^{3} \mathrm{P}_{1}\right)+1,08 \mathrm{eV} .
\end{aligned}
$$

En effet, pour ce dernier processus, le taux de transfert d'orientation est élevé $\left(\alpha^{(1)}=0,42 \pm 0,10\right)$ et le taux de transfert d'alignement a été trouvé positif $\left(\alpha^{(2)}=0,47 \pm 0,11\right)$ [3]. Ces résultats sont justifiés qualitativement à partir d'un modèle de collision dans lequel les collisions frontales jouent un rôle prépondérant (faible rotation de l'axe moléculaire au cours de la collision). Par contre une rotation importante de l'axe de la collision conduirait à des résultats différents et pourrait expliquer les résultats obtenus ici. Cependant, cela suppose que la section efficace de transfert de population $\sigma^{(0)}$ des processus (1) et (2) est beaucoup plus grande que celle du processus (7). Nous entreprenons actuellement la mesure de $\sigma^{(0)}$.

\section{Bibliographie}

[1] Kraulinya, E. K. and Arman, M. G., Opt. Spectrosc. 26 (1969) 285.

[2] Morozov, E. N. and SosinskiI, M. L., Opt. Spectrosc. 26 (1969) 282.

[3] Cheron, B., J. Physique 36 (1975) 17 et 29.

[4] Cheron, B., Cremer, G., Lecler, D., Saintout, L., J. Physique Lett. 35 (1974) L-247. 